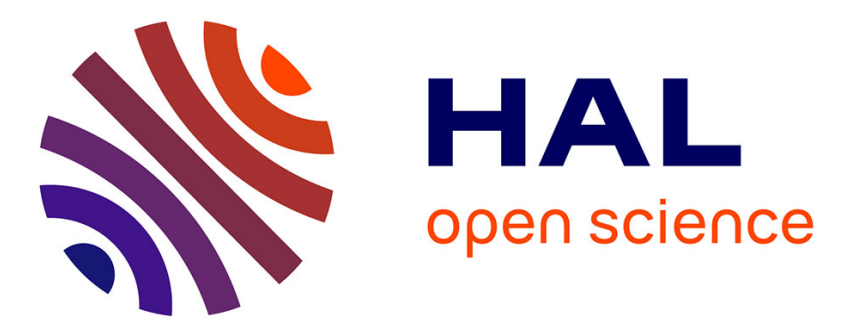

\title{
Quantifier l'influence totale de la famille d'origine sur le devenir scolaire et professionnel des individus
}

Béatrice Boutchenik, Clotilde Coron, Sébastien Grobon, Céline Goffette, Louis-André Vallet

\section{- To cite this version:}

Béatrice Boutchenik, Clotilde Coron, Sébastien Grobon, Céline Goffette, Louis-André Vallet. Quantifier l'influence totale de la famille d'origine sur le devenir scolaire et professionnel des individus. Economie et Statistique / Economics and Statistics, 2015, 477 (1), pp.5 - 23. hal-01520293

HAL Id: hal-01520293

https://hal-sciencespo.archives-ouvertes.fr/hal-01520293

Submitted on 10 May 2017

HAL is a multi-disciplinary open access archive for the deposit and dissemination of scientific research documents, whether they are published or not. The documents may come from teaching and research institutions in France or abroad, or from public or private research centers.
L'archive ouverte pluridisciplinaire HAL, est destinée au dépôt et à la diffusion de documents scientifiques de niveau recherche, publiés ou non, émanant des établissements d'enseignement et de recherche français ou étrangers, des laboratoires publics ou privés. 


\title{
Quantifier l'influence totale de la famille d'origine sur le devenir scolaire et professionnel des individus
}

\author{
Béatrice Boutchenik, Clotilde Coron, Sébastien Grobon, \\ Céline Goffette et Louis-André Vallet *
}

Les travaux visant à mesurer l'influence familiale sur les parcours scolaires et professionnels reposent principalement sur des données relatives à un individu et ses ascendants. Celles-ci réduisent l'influence du milieu familial à l'effet des caractéristiques mesurées dans les enquêtes, tels le diplôme et la profession de chacun des deux parents. La part de variance expliquée par ces variables fournit une estimation basse et biaisée de l'influence de la famille d'origine, réduite à ces seules variables explicatives : elle ignore l'impact éventuel des aspects familiaux inobservés ou inobservables.

Cet article s'affranchit de cette limite en s'inscrivant dans la tradition des modèles de fratrie (sibling models). Il utilise les données sur les différents membres d'une fratrie disponibles dans deux enquêtes : l'enquête sur la formation et la qualification professionnelle (FQP) de l'Insee (2003) et l'enquête sur le niveau intellectuel des enfants d'âge scolaire de l'Ined (1965). Observer et modéliser les parcours d'individus ayant connu une socialisation familiale similaire permet de prendre en compte toutes les formes de transmission communes aux frères et sœurs. L'influence familiale est ainsi considérée au sens large en tant qu'effet de l'ensemble du milieu social d'origine. Dans la mesure où elle amène les parcours des membres de la fratrie à se ressembler, nous la qualifions de « cohésive ».

Les données de l'enquête FQP montrent que l'influence familiale cohésive explique la moitié de la variabilité des niveaux d'éducation entre individus, et un tiers de la variabilité des niveaux de profession. Au sein de la part relevant de l'influence familiale

Rappel :

Les jugements et opinions exprimés par les auteurs n'engagent qu'eux mêmes, et non les institutions auxquelles ils appartiennent, ni a fortiori l'Insee. cohésive, la moitié seulement est expliquée par les caractéristiques du milieu familial observées dans l'enquête. Par ailleurs, l'influence familiale est plus forte pour les paires fraternelles de même sexe, lorsque l'écart d'âge entre frères et sœurs est limité, ou que les diplômes des parents sont plus homogènes.

Codes JEL : J62, I24, I26, Z13.

Mots-clés : influence familiale, déterminismes sociaux, mobilité sociale, modèles de fratrie, sibling models, inégalités scolaires, rendements de l'éducation, socialisation primaire, modèles Mimic.

* Cet article est issu d'une recherche menée dans le cadre d'un groupe de travail de l'Ensae. Béatrice Boutchenik, Clotilde Coron et Sébastien Grobon étaient alors étudiants à l'Ensae. Céline Goffette et Louis-André Vallet étaient membres du Laboratoire de Sociologie Quantitative (Crest-Grecsta).

Les auteurs remercient Françoise Moreau de l'Ined pour son aide précieuse apportée lors de la mise en forme des données de l'enquête nationale de 1965 sur le niveau intellectuel des enfants d'âge scolaire. Ils remercient également les deux rapporteurs anonymes de la revue pour leurs remarques et suggestions. 
D epuis plusieurs décennies, les sociologues cherchent à mesurer le degré auquel le statut social des individus est influencé par celui de leur famille. Les travaux quantitatifs portant sur la transmission familiale intergénérationnelle $\mathrm{du}$ statut utilisent classiquement des données portant sur un individu et au moins l'un de ses ascendants. Les critiques formulées à l'égard de ces travaux ont progressivement amené à en renouveler l'approche, en tirant parti de données portant sur plusieurs membres d'une fratrie.

\section{Des données sur l'origine sociale aux données de fratrie : une brève histoire des modèles de fratrie}

L'étude de la mobilité sociale reposait initialement sur l'analyse de tableaux croisant la catégorie sociale du fils et celle de son père (tables de mobilité). Par la suite, les modèles d'acquisition du statut (status attainment), développés dans le cadre de la sociologie américaine de la mobilité sociale et dont l'ouvrage de Blau et Duncan The American Occupational Structure (1967) constitue l'exemple canonique de mise en œuvre, ont renouvelé l'étude de la mobilité sociale. Ces modèles visaient à étudier l'effet direct et indirect (transitant par l'éducation) de l'origine sociale sur le statut professionnel. Leur apparition est allée de pair avec l'utilisation d'échelles numériques pour les variables d'intérêt que sont le niveau d'éducation et le statut professionnel, qui ont remplacé les variables catégorielles utilisées jusqu'alors. Mais, quel que soit le niveau de détail atteint pour les déterminants familiaux de l'éducation et de la profession, ces modèles traditionnels ne peuvent rendre compte que partiellement de l'influence familiale sur les parcours individuels. C'est le sens de la critique adressée par Bowles aux modèles de status attainment (1972) : parmi les éléments transmis par la famille et influant sur les parcours des individus, certains ne peuvent être mesurés que de façon approximative, ou ne peuvent tout simplement pas être observés dans le cadre d'une enquête statistique.

Dès la même année, Duncan, Featherman et Duncan montrent l'existence d'une corrélation entre les niveaux scolaires des frères et sœurs (1972). La Wisconsin Longitudinal Study, une enquête américaine longitudinale de grande ampleur comportant 10000 individus, intègre des variables renseignant les parcours scolaires des frères et sœurs. Hauser et Sewell (1977) estiment que l'emploi de ces données de fratrie rend possible une meilleure analyse de l'influence familiale, et donc des inégalités sociales. Ces observations donnent lieu au développement des modèles de fratrie (sibling models), qui se fondent sur l'utilisation de données relatives aux frères et sœurs pour estimer de façon plus précise les rôles respectifs du milieu social d'origine et de la famille, de l'école, et des caractéristiques individuelles dans la réussite socioprofessionnelle. L'observation de la réussite scolaire et professionnelle des frères et sœurs permet de mesurer ce qui provient de l'influence familiale (qui est d'autant plus grande que les parcours se ressemblent dans la fratrie), et de la distinguer de ce qui provient d'une différenciation individuelle. Plus récemment, cette tradition a été renouvelée par des sociologues néerlandais (De Graaf (1986), Sieben (2001), Sieben et De Graaf (2003)) qui systématisent l'utilisation de données et de modèles de fratrie en Europe pour mesurer l'influence cohésive de la famille et comparer l'importance du milieu d'origine dans différents pays. La France n'a pas pu être incluse dans ces analyses du fait de la rareté de données de fratries portant sur la réussite scolaire et socioprofessionnelle jusqu'en $2003^{1}$. Les approches classiques de la mobilité sociale, largement utilisées en France, gagnent pourtant à être articulées avec les modèles de fratrie pour analyser de manière plus complète la transmission familiale intergénérationnelle $\mathrm{du}$ statut social. Comme le soulignent Monso et Thévenot (2008), l'information relative à un frère ou une sœur renseigne sur un « destin social familial » : un même parcours social intergénérationnel peut être interprété différemment selon le parcours des frères et sœurs de l'individu concerné.

\section{L'influence familiale définie par les modèles de fratrie incorpore toutes les formes de socialisation communes aux membres de la fratrie}

L'influence familiale mesurée par les modèles de fratrie inclut non seulement l'effet des

1. En France, Bernard Zarca s'interroge sur la mobilité sociale différentielle des frères (1995a) et des sœurs (1995b), dans une étude "à l'intersection de la mobilité sociale et de celle des rapports entre germains ". II exploite l'enquête de l'Ined de 1976 sur les réseaux familiaux, portant sur un échantillon de 2000 individus de 45 à 64 ans pour lesquels on dispose d'informations sur certains membres de la fratrie. Cette enquête porte sur des cohortes beaucoup plus anciennes que celles analysées par Sieben et De Graaf dans leur étude comparative. Zarca montre le poids de l'héritage familial sur les parcours des frères et des sœurs, dont résulte une "complémentarité des destins professionnels et sociaux ". II met également en évidence des phénomènes de différenciation au sein de la fratrie, qui s'expriment notamment par un « incontestable avantage de l'aîné des frères sur son frère benjamin dans les familles des classes aisées ». 
caractéristiques familiales mesurables, mais également celui de toutes les caractéristiques inobservables que les frères et sœurs peuvent partager. Ces caractéristiques sont de trois ordres. Premièrement, l'influence familiale s'exerce verticalement, des parents vers les enfants, et les caractéristiques familiales inobservables englobent donc l'hérédité au sens large, génétique ${ }^{2}$, culturelle et sociale. Deuxièmement, l'influence familiale a également une dimension horizontale, puisqu'elle recouvre aussi l'influence réciproque entre frères et sœurs, que Hauser et Wong (1989) ont modélisée en s'intéressant aux configurations de genre et d'âge dans la fratrie. Enfin, ce facteur intègre l'influence de l'environnement commun aux frères et sœurs (Griliches, 1979) : quartier, qualité de l'école fréquentée, influence des pairs, etc. Si ces éléments dépassent le cadre strict de la famille, on peut toutefois considérer qu'ils relèvent de l'influence familiale, dans la mesure où le choix du quartier de résidence, par exemple, provient de décisions prises par les parents. Les analyses de fratrie permettent ainsi de mesurer une influence familiale qui a été qualifiée de totale (Sieben, 2001).

\section{En utilisant la ressemblance dans la fratrie, on mesure un impact de l'éducation sur la réussite professionnelle net du biais lié à l'origine familiale}

C'est dans la perspective d'isoler l'influence propre de l'éducation de celle de l'origine sociale sur le statut professionnel que Bowles (1972) a effectué sa critique des status attainment models initiaux. On rejoint ici une problématique centrale en économie de l'éducation, qui est celle du rendement de l'éducation que l'on cherche à mesurer en évitant un biais de variable omise dû à l'influence des capacités (ability) à la fois sur l'éducation et le salaire. Griliches et Mason (1972) ont par exemple inclus dans la régression initiale des contrôles comme les résultats à des tests de QI ou des variables relatives au style d'éducation parental : l'effet de l'éducation mesuré diminue alors d'un peu plus de $10 \%$. Les modèles de ressemblance dans la fratrie permettent de mesurer l'effet de l'éducation sur le statut professionnel en contrôlant l'influence familiale exercée de façon simultanée sur ces deux variables.

La présentation des résultats de notre analyse suit le cheminement de ces différentes problématiques : dans un premier temps, après avoir présenté les modèles permettant de mesurer l'influence familiale cohésive (notamment sur la réussite scolaire et professionnelle), cette influence est quantifiée et comparée à l'influence familiale mesurable grâce aux seules caractéristiques familiales habituellement observées. La façon dont l'influence familiale cohésive peut varier selon certaines caractéristiques (sexe des binômes considérés, écart d'âge...) est ensuite étudiée. À la suite de cette analyse principale de l'influence familiale cohésive, celle-ci est utilisée dans l'étude de l'effet de l'éducation sur la profession, en tant qu'outil permettant un meilleur contrôle de l'origine sociale et de l'appartenance familiale. Enfin, nous précisons l'interprétation qui peut être faite de l'influence familiale cohésive mesurée par la ressemblance dans la fratrie, relativement à une analyse classique en termes de reproduction ou de mobilité sociale.

\section{Les modèles Mimic permettent de mesurer l'influence d'un facteur familial englobant mesuré comme variable latente}

Les modèles de fratrie exploités ici relèvent de la catégorie des modèles Mimic (Multiples Indicateurs Multiples Causes) (cf. encadré 1), construits autour d'un facteur latent perceptible à travers plusieurs indicateurs. Dans le cadre de l'analyse de fratrie, la variable latente se comprend comme un « facteur familial ». Elle n'est pas observable mais peut être mesurée à partir d'indicateurs correspondant ici à nos variables d'intérêt qui sont le niveau d'éducation ou le statut professionnel. Dans un schéma causal, ce facteur familial est ainsi déterminé par des caractéristiques observables mais également inobservables de la famille, et influe sur une variable d'intérêt donnée, renseignée pour les différents membres de la fratrie, et qui peut également être influencée par des caractéristiques individuelles (cf. figure I).

Par construction statistique, l'ensemble des effets des caractéristiques familiales sur le niveau d'éducation ou le statut professionnel des enfants passe par la variable latente, inférée en s'appuyant sur la ressemblance

2. Les recherches modernes en psychologie et en épigénétique montrent que la séparation entre l'influence des gènes et celle de l'environnement (intra- et extrafamilial) perd son sens puisque les gènes s'expriment différemment selon les stimulations qu'ils reçoivent. Cela invite, plutôt que d'essayer d'isoler l'effet propre des gènes, à le mesurer en interaction avec une hérédité au sens large comme c'est le cas dans la présente étude. 
entre frères et sœurs. Empiriquement, l'influence cohésive de la famille est alors définie comme la part de la variance des parcours qui différencie les familles entre elles (variance inter-familles). La part de variance intra-familles, c'est-à-dire celle qui mesure les différences de parcours au sein de la même famille, reflète au contraire la différenciation au sein de la fratrie. C'est donc sur cette décomposition de la variabilité des parcours entre fratries et au sein des fratries que repose la mesure de l'influence familiale cohésive.

\section{Encadré 1}

\section{COMMENT ESTIME-T-ON UN MODĖLE MIMIC ?}

Les modèles Mimic sont un type de modèles à équations structurelles. Jöreskog et Goldberger (1975) détaillent la spécification et l'estimation d'un modèle Mimic comprenant une seule variable latente, de même que les modèles estimés ici. L'estimation d'un tel modèle Mimic requiert de spécifier l'ensemble des influences existant entre les $\mathrm{N}$ variables, que celles-ci soient manifestes ou latentes. Le modèle est ainsi décrit dans notre cas par les équations suivantes, qui formalisent les relations illustrées par la figure I:

$Y_{\mathrm{L}}=\alpha_{\mathrm{L}}+\beta_{\mathrm{L}} \cdot X_{\mathrm{L}}+\varepsilon_{\mathrm{L}}$

$Y_{\mathrm{R}}=\alpha_{\mathrm{R}}+\beta_{\mathrm{R}} \cdot X_{\mathrm{R}}+\gamma_{\mathrm{R}} \cdot Y_{\mathrm{L}}+\varepsilon_{\mathrm{R}}$

$Y_{\mathrm{FS}}=\alpha_{\mathrm{FS}}+\beta_{\mathrm{FS}} \cdot X_{\mathrm{FS}}+\gamma_{\mathrm{FS}} \cdot Y_{\mathrm{L}}+\varepsilon_{\mathrm{FS}}$

Avec $Y_{L}$ la variable latente ou "facteur familial ", $X_{\llcorner}$les caractéristiques observables communes à la fratrie (par exemple la profession des parents), $Y_{\mathrm{B}}$ et $Y_{\mathrm{FS}}$ les variables d'intérêt caractérisant respectivement le répondant et son frère ou sa sœur tiré(e) au hasard (par exemple leur niveau d'études), $X_{R}$ et $X_{\mathrm{FS}}$ les caractéristiques individuelles de ces derniers (notamment le sexe, la position dans la fratrie), et les différents $\alpha$ et $\varepsilon$ des constantes et termes d'erreur associés à chaque équation.

On impose par ailleurs dans notre spécification l'égalité des coefficients $\alpha_{R}$ et $\alpha_{F S}$, celle de $\beta_{R}$ et $\beta_{F S}$, de $\gamma_{R}$ et $\gamma_{F S}$, et enfin l'égalité de la variance de $\varepsilon_{R}$ et $\varepsilon_{\mathrm{FS}}$. Cela signifie que les répondants et frères/sœurs jouent des rôles symétriques dans les équations, ce qui revient à supposer que le facteur familial latent a une influence identique pour chacun d'eux et que les variables individuelles des deux membres de la fratrie considérés influencent avec la même intensité la variable d'intérêt.

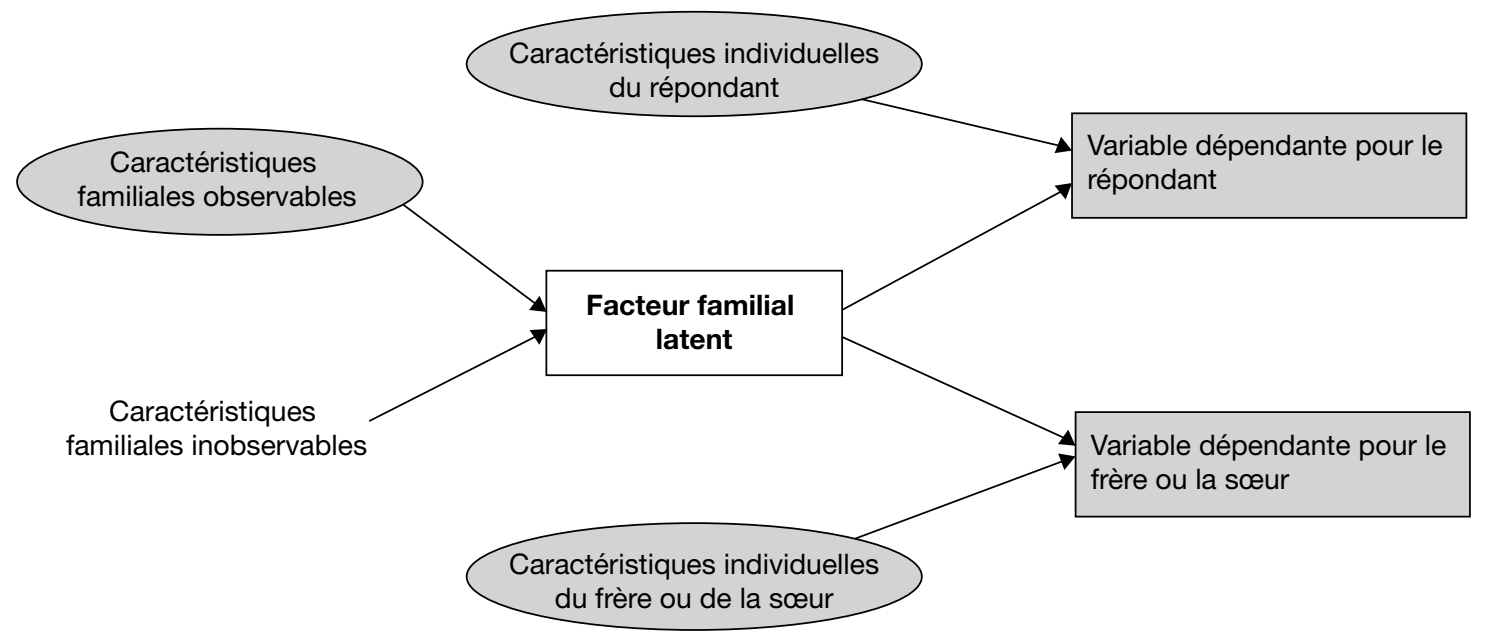

Lecture : les flèches représentent les relations de causalité supposées entre les différentes variables, présentes dans l'enquête ou estimées. La variable dépendante, renseignée pour le répondant et un membre de sa fratrie, permet l'estimation du facteur familial latent. Les facteurs non observables sont indiqués en blanc et les facteurs observables en gris. À partir des caractéristiques familiales présentes dans l'enquête, on estime en creux l'ampleur de l'influence des caractéristiques familiales inobservables. 


\section{Le milieu familial explique la moitié des différences de niveau d'éducation entre individus}

Le modèle Mimic tel que décrit plus haut est mis en œuvre sur les données de l'enquête Formation et qualification professionnelle de
2003 (cf. encadré 2). Le facteur familial latent explique $54,3 \%$ de la variabilité du nombre d'années d'études mesuré selon l'échelle modale (cf. tableau 1 ; se reporter à l'encadré 2 et au tableau A de l'annexe pour la définition des échelles). Le même ordre de grandeur -50 à $60 \%$ - est mis en évidence par Sieben (2001)

Encadré 2

\section{LES PARCOURS SCOLAIRES ET PROFESSIONNELS DANS LA FRATRIE, VUS À TRAVERS L'ENQUÊTE FQP 2003}

\section{Champ de l'enquête Formation et qualification} professionnelle de $\mathbf{2 0 0 3}$ et de l'échantillon retenu

Le champ de l'enquête FQP 2003 est constitué de 39312 personnes ayant entre 18 et 65 ans au moment de l'enquête, tirées au sort dans l'échantillon de logements de l'Insee. L'échantillon est restreint aux individus âgés de plus de 25 ans et dont les parents résidaient en France au moment de la fin de leurs études, de façon à ne conserver que des individus ayant très probablement fini leurs études et suivi celles-ci au moins pour partie dans le système scolaire français. Cela permet d'obtenir un ensemble de 29954 individus. On limite également l'échantillon aux individus ayant au moins un frère ou une sœur, les enfants uniques ne pouvant pas être inclus dans les modèles Mimic (voir l'encadré 3 dans lequel nous nous interrogeons sur l'existence d'un biais lié à cette restriction). De plus, la variable d'éducation ou la variable de profession doivent être renseignées à la fois pour le répondant et pour son frère ou sa sœur. Au final, l'étude porte sur un échantillon de 25259 paires fraternelles pour l'analyse du niveau d'éducation, et de 23181 paires fraternelles pour l'analyse du statut professionnel.

Des informations sur un membre de la fratrie tiré au hasard, renseignées par le répondant

Une des spécificités de l'enquête tient à ce que des informations sont fournies par le répondant sur le parcours scolaire et professionnel d'un frère ou une sœur tiré(e) au hasard dans sa fratrie, le cas échéant. Le fait qu'un membre de la fratrie soit tiré au hasard garantit une absence de biais relatif au choix du frère ou de la sœur sur lequel le répondant souhaiterait (ou serait en mesure de) fournir certaines informations. Néanmoins, ce mode de déclaration des variables relatives au membre de la fratrie tiré au hasard peut conduire à des imprécisions, si le répondant ne possède pas suffisamment d'information relative à celui-ci. Ainsi, on constate que les structures de diplôme et de profession ne sont pas entièrement symétriques pour le groupe des répondants et celui des frères et sœurs tirés au hasard : ce dernier ensemble comporte notamment une moins grande proportion de réponses "Aucun diplôme ou CEP » que celle observée chez les répondants $(25,4 \%$ contre $29,0 \%$ ) et au contraire une proportion supérieure de "CAP, BEP ou autre diplôme de ce niveau » $(30,6 \%$ contre $26,6 \%)$. Ce biais de déclaration pourrait influer sur les résultats obtenus, si par exemple une partie des répondants tendait à renseigner un parcours similaire au leur lorsque le parcours du membre de leur fratrie tiré au hasard n'est pas connu précisément, ce qui impliquerait un rapprochement artificiel des parcours. L'erreur d'estimation globalement liée à de telles erreurs de déclaration est néanmoins jugée négligeable par Sieben (2001), à la suite des travaux de Van Eijck (1996).

Appréhender les parcours par le nombre d'années d'études et le score de statut social

Dans l'enquête $F Q P$, deux aspects de la proximité des parcours de membres d'une même paire fraternelle peuvent être appréhendés : proximité en termes d'éducation, et proximité en termes de profession. II est nécessaire de disposer de variables numériques pour ces deux dimensions, les modèles Mimic n'étant pas applicables à des variables d'intérêt catégorielles.

Concernant l'éducation, le diplôme le plus élevé est converti en nombre d'années d'études. L'enquête ne renseigne pas le nombre effectif d'années d'études pour le membre de la fratrie tiré au hasard, ce qui rend nécessaire la construction d'une échelle associant à chaque diplôme un nombre d'années d'études. II serait souhaitable de pouvoir associer à chaque diplôme le nombre normal d'années nécessaires à son obtention - pour un élève n'ayant jamais redoublé ni sauté une classe. C'est la stratégie utilisée en particulier par Ganzeboom et Treiman (1993). Cela est toutefois impossible ici, d'une part car certaines catégories de diplôme regroupent plusieurs nombres d'années requises différents, d'autre part parce que ces nombres normaux ont pu varier pendant la longue période que couvre l'enquête. Une première échelle, appelée ici échelle modale, a été construite en associant, à chaque catégorie détaillée de diplôme maximal obtenu, le mode du nombre d'années d'études effectivement suivies par les individus possédant ce diplôme, c'est-à-dire la différence entre l'âge à la fin des études, et l'âge de six ans. Une seconde échelle, appelée ici échelle médiane, s'appuyant cette fois sur la médiane du nombre d'années d'études pour les individus possédant au maximum un diplôme donné a aussi été construite. Les résultats obtenus avec les deux échelles sont très proches, et pourront sauf mention contraire être commentés indifféremment.

Pour la profession, l'échelle élaborée par Chambaz, Maurin et Torelli (1998) associant aux différentes PCS des scores de statut social, obtenus en s'appuyant sur les classements subjectifs de 122 professions 
Encadré 2 (suite)

effectués par 3600 individus environ, permet de disposer d'une proxy quantitative de la variable catégorielle de la PCS. Cette échelle existe sous deux formes. L'échelle non linéaire est construite sur l'hypothèse que les scores subjectifs attribués par les individus aux professions sont des indications qualitatives permettant de restituer uniquement l'ordre des professions en termes de statut. Dans le cas de l'échelle linéaire, ces scores sont considérés comme des quantités reflétant la valeur sociale associée à chaque profession. D'après les auteurs, le modèle linéaire donne des résultats très proches de ceux du modèle non linéaire (le détail des échelles utilisées figure p. 200 de l'article de Chambaz et al. Voir le tableau $B$ en annexe pour une présentation simplifiée). C'est également le cas pour les résultats présentés ici, et seuls ceux portant sur l'échelle non-linéaire seront présentés.

Tableau 1

Coefficients standardisés et parts de variance expliquées d'un modèle Mimic expliquant le nombre d'années d'études, incluant caractéristiques familiales puis caractéristiques individuelles et familiales

\begin{tabular}{|c|c|c|}
\hline & Échelle modale & Échelle médiane \\
\hline \multicolumn{3}{|l|}{ Déterminants de la variable latente - variables familiales } \\
\hline Score de la PCS du père & $0,198\left(^{\star * \star}\right)$ & $0,200\left(^{* * *}\right)$ \\
\hline Score de la PCS de la mère & $0,112\left(^{\star \star \star}\right)$ & $0,119\left(^{(\star \star}\right)$ \\
\hline Père inactif n'ayant jamais travaillé & $-0,005$ & $-0,005$ \\
\hline Mère inactive n'ayant jamais travaillé & $-0,050\left({ }^{* \star \star}\right)$ & $-0,050\left({ }^{* \star \star}\right)$ \\
\hline Score de la PCS du grand-père maternel & $\left.0,064{ }^{(* \star}\right)$ & $0,066\left(^{* \star *}\right)$ \\
\hline Score de la PCS du grand-père paternel & $0,024\left(^{* \star *}\right)$ & $0,026\left(^{* \star \star}\right)$ \\
\hline PCS du grand-père maternel manquante & $-0,091(\star \star \star)$ & $-0,089\left({ }^{\star \star \star}\right)$ \\
\hline PCS du grand-père paternel manquante & $-0,082\left({ }^{\star \star \star}\right)$ & $-0,082\left({ }^{\star \star \star}\right)$ \\
\hline Nombre d'années d'études du père & $0,197\left(^{\star \star \star}\right)$ & $0,202\left(^{* \star \star}\right)$ \\
\hline Nombre d'années d'études de la mère & $0,137\left(^{\star \star \star}\right)$ & $0,142\left(^{* \star \star}\right)$ \\
\hline Nombre d'enfants dans la fratrie & $-0,176\left({ }^{\star \star \star}\right)$ & $-0,179\left({ }^{\star \star \star}\right)$ \\
\hline Interaction avec une indicatrice « père cadre ou chef d'entreprise » & $0,028\left(^{* * *}\right)$ & $0,034\left(^{* * *}\right)$ \\
\hline Interaction avec une indicatrice « père ouvrier ou employé » & $-0,057(\star \star \star)$ & $-0,049\left({ }^{\star \star \star}\right)$ \\
\hline Divorce ou séparation des parents pendant les études & $-0,018\left(^{\star \star}\right)$ & $-0,021\left({ }^{\star \star \star}\right)$ \\
\hline Parents résidant en Île-de-France à la fin des études & $\left.0,021{ }^{\star \star \star}\right)$ & $0,022\left(^{* \star \star}\right)$ \\
\hline Taux de chômage dans la région des parents à la fin des études & $-0,004$ & $-0,005$ \\
\hline Score PCS manquant pour le père (hors inactif, jamais travaillé) & $-0,011$ & $-0,012(*)$ \\
\hline Score PCS manquant pour la mère (hors inactive, jamais travaillé) & $-0,023\left({ }^{\star * *}\right)$ & $-0,020\left({ }^{* \star *}\right)$ \\
\hline Part de variance expliquée par le facteur familial latent (en \%) & 54,3 & 55,6 \\
\hline $\begin{array}{l}\text { Part de variance du facteur familial latent expliquée par les variables familiales observables } \\
\text { (en \%) }\end{array}$ & 49,3 & 51,0 \\
\hline \multicolumn{3}{|l|}{ Déterminants de la variable dépendante - variables individuelles } \\
\hline Sexe féminin & $0,028\left(^{\star * \star}\right)$ & $0,026\left(^{\star \star *}\right)$ \\
\hline Aîné & $0,028\left(^{\star * \star}\right)$ & $0,031\left(^{* \star \star}\right)$ \\
\hline Benjamin & $0,017\left(^{\star \star \star}\right)$ & $0,015(* \star)$ \\
\hline Âge de la mère à la naissance & $0,064(* \star *)$ & $0,065\left({ }^{* \star *}\right)$ \\
\hline Effet du facteur familial sur le nombre d'années d'études effectué & $0,737\left(^{\star \star *}\right)$ & $0,745\left(^{\star * \star}\right)$ \\
\hline Part de variance expliquée par le facteur familial latent et les variables individuelles (en \%) & 54,8 & 56,1 \\
\hline
\end{tabular}

Lecture: Bien que l'on ait imposé l'égalité des coefficients entre répondant et frère/sœur dans les équations expliquant le nombre d'années d'études (cf. encadré 1), ces contraintes s'appliquent aux coefficients non-standardisés et non aux coefficients standardisés. Les variances des caractéristiques individuelles pouvant varier entre les répondants et l'ensemble des frères ou sœurs tirés au hasard, les coefficients standardisés des équations concernées ne sont pas nécessairement égaux. Toutefois ils varient très peu (au maximum de 0,002) entre les deux équations.

Afin de traiter les valeurs manquantes concernant la PCS de la mère, le score moyen parmi les autres mères de l'échantillon a été impute aux observations concernées (12 121 dans l'échantillon initial). Sont alors également incluses dans le modèle une variable indicatrice concernant les mères inactives ou n'ayant jamais travaillé, ainsi qu'une indicatrice désignant les autres cas de valeurs manquantes. Les valeurs manquantes pour les grands-pères sont traitées par deux variables indicatrices (grand-père paternel et grand-père maternel) couvrant toutes les valeurs manquantes, et par deux variables indicatrices pour les parents (père et mère).

Une augmentation du niveau d'études du père de l'enquêté à hauteur d'un écart-type augmenterait de 0,197 écart-type le facteur familial, qui aurait lui-même un effet positif (+0,737 écart-type) sur le nombre d'années d'études effectué par l'enquêté. La part de variance du niveau d'études expliquée par le facteur familial latent, qui mesure l'influence familiale, est de 54,3\%.

${ }^{* * *}$ : significatif au seuil de $1 \% ;{ }^{* *}$ : significatif au seuil de $5 \% ;{ }^{*}$ significatif au seuil de $10 \%$.

Champ : personnes âgées de plus de 25 ans résidant en France métropolitaine, dont les parents résidaient en France au moment de la fin de leurs études.

Source : enquête FQP 2003, Insee. 
sur un échantillon de 11 pays. L'influence familiale cohésive permet ainsi d'expliquer plus de la moitié de la diversité des parcours scolaires ; en creux, moins de la moitié de celle-ci est due à la différenciation au sein de la fratrie.

L'inclusion de caractéristiques individuelles ne permet d'augmenter le pouvoir explicatif du modèle que faiblement, pour moins de $1 \%$. Les coefficients standardisés concernant les caractéristiques individuelles sont cohérents avec la littérature existante. Le fait d'être une fille fait par exemple significativement augmenter le nombre d'années d'études toutes choses égales par ailleurs. De même, il existe non seulement un avantage au fait d'être l'aîné(e), mais également un léger avantage au fait d'occuper le dernier rang dans la fratrie (bien que la différenciation selon le rang dans la fratrie reste faible par rapport à l'influence familiale commune à tous les frères et sœurs).

Le modèle incorpore par ailleurs l'ensemble des caractéristiques familiales disponibles dans l'enquête $F Q P$ (se reporter au tableau $\mathrm{C}$ de l'annexe pour des statistiques descriptives sur les variables explicatives). L'inclusion des variables familiales conduit à distinguer ce qui, dans le facteur familial latent, dépend de ces caractéristiques familiales observables, de ce qui correspond à des caractéristiques familiales inobservables impactant de la même manière l'éducation des deux membres de la fratrie: 49,3\% de la variance du facteur familial latent est expliquée par les caractéristiques familiales observées, dans le cas de l'échelle modale.

Les déterminants les plus importants du facteur familial sont le score de la PCS et le nombre d'années d'études du père, le nombre d'enfants dans la fratrie, puis le nombre d'années d'études et le score de la PCS de la mère. Le nombre d'enfants dans la fratrie est négativement corrélé avec le facteur familial. Cette relation est potentiellement endogène, des éléments de contexte familial pouvant jouer à la fois sur la taille de la fratrie et sur la réussite scolaire ou la destinée sociale. La relation est par ailleurs d'autant moins forte que la catégorie sociale est élevée, comme le montrent les termes d'interaction du nombre d'enfants dans la fratrie avec la catégorie sociale du père. Cet effet a été observé par exemple par Vallot (1973) dans le cas de la performance scolaire.

Ainsi, même l'inclusion d'un ensemble très complet de variables familiales observables ne permet de rendre compte que de la moitié de l'influence cohésive exercée par l'appartenance familiale sur le nombre d'années d'études.

Encadré 3

\section{L'EXCLUSION DES ENFANTS UNIQUES CONDUIT-ELLE À UN BIAIS ?}

L'analyse de fratrie étant restreinte aux répondants ayant au moins un frère ou une sœur, il est important de souligner d'éventuelles différences qui pourraient exister entre les enfants uniques et les autres. II y a trois catégories de répondants dans le cadre de l'enquête FQP : ceux qui ont des frères et sœurs pour lesquels on possède la variable du diplôme et qui représentent $88,1 \%$ de l'échantillon, ceux qui sont enfants uniques $(10,1 \%)$, et enfin ceux qui ont des frères et sœurs dont on ne connaît pas le diplôme (1,8 \%).

Les enfants ayant un père artisan, commerçant ou chef d'entreprise, cadre, employé ou exerçant une profession intermédiaire, sont relativement plus nombreux parmi les enfants uniques que parmi les répondants issus de fratries, alors que l'inverse est vrai des enfants d'agriculteurs et d'ouvriers. Une étude sur la catégorie socioprofessionnelle des mères d'enfants uniques conduit aux mêmes résultats. Ceux-ci sont en accord avec les observations démographiques de fécondité différentielle selon les PCS (Merllié et Monso, 2007).

Les enfants uniques ont en moyenne des diplômes plus élevés que les autres. Cela s'explique tout d'abord par leur origine sociale différente, soulignée ci-dessus. Néanmoins, à PCS du père donnée, la différence entre enfants uniques et enfants issus de fratrie reste présente, quoique moindre. Cela confirme un autre aspect de l'analyse de Merllié et Monso : à origine sociale donnée, les différences de destinées sociales sont liées à la taille de la famille d'origine.

Un travail d'analyse mené sur les données de l'enquête $F Q P$ fait ressortir que la proximité dans la fratrie en termes de nombre d'années d'études, donc l'importance de l'influence familiale telle que mesurée ici, décroît lorsque la taille de la fratrie augmente. Prolonger cette tendance (qui par définition n'est mesurable que pour des fratries) aux enfants uniques conduirait donc à penser que leur parcours est plus largement déterminé par leur appartenance familiale que pour les autres fratries, que cet effet soit endogène ou non. II pourrait ainsi exister un biais potentiel associé au fait d'exclure les enfants uniques, exclusion inhérente au principe même de l'analyse de fratries. Ce problème a également été soulevé par Sieben (2001), qui estime toutefois qu'il serait négligeable. 
On retrouve ici encore l'ordre de grandeur obtenu par Sieben (2001) pour l'échantillon de pays étudiés, pour lesquels $45 \%$ en moyenne de la variance du facteur familial est expliquée par les caractéristiques observables. Les caractéristiques observées n'expliquent donc que pour moitié le facteur familial, qui lui-même explique plus de la moitié des disparités des niveaux d'éducation.

\section{L'influence familiale reste constante au cours du temps}

Les estimations qui ont été réalisées rassemblent dans un même échantillon des individus ayant grandi à des périodes pouvant être éloignées dans le temps. En couplant ce fait avec l'allongement de la durée des études au fil des générations, on pourrait mesurer une variance inter-familles du nombre d'années d'études ne correspondant pas au phénomène que nous souhaitons approcher (l'influence cohésive de la famille au sein d'une génération donnée), artificiellement gonflée par les écarts d'éducation entre des enfants appartenant à des fratries ayant grandi à des périodes éloignées de plus de quarante ans. Cela conduit à estimer les modèles Mimic cohorte par cohorte pour éviter de capter une trop grande partie d'évolutions structurelles dans la variance inter-familles. Dans le cas de la variable médiane pour le nombre d'années d'études, 54,8\% de la variance est expliquée par le facteur familial pour les 34 ans et moins, $53,5 \%$ pour les 35 à 44 ans, 53,1\% pour les 45 à 54 ans et enfin $51,3 \%$ pour les 55 ans et plus ${ }^{3}$. L'estimation du modèle Mimic sur l'ensemble de la population ne conduit donc pas à surestimer fortement l'influence familiale.

Cette analyse pourrait permettre par ailleurs de mettre en évidence une éventuelle évolution de l'influence familiale sur le nombre d'années d'études au cours du temps. Il apparaît qu'aucune des différences de coefficients entre deux cohortes consécutives n'est significative. Seul l'écart entre la plus jeune et la plus ancienne cohorte est significatif, au seuil de $5 \%$. Il ne l'est toutefois plus dès lors qu'on utilise la variable modale. La tendance temporelle observée, qui est celle d'une influence familiale commune aux frères et sœurs de plus en plus élevée dans les générations récentes, ne semble donc pas assez marquée pour que l'on puisse en tirer de conclusions fortes. Elle va en tout cas à l'encontre de l'hypothèse d'une individualisation croissante des choix scolaires et professionnels dans la famille au fil des cohortes.

Une source statistique plus ancienne, l'enquête sur le niveau intellectuel des enfants d'âge scolaire de l'Ined (1965), permet par ailleurs de quantifier l'influence familiale en utilisant non plus le nombre d'années d'études, mais les résultats de tests psycho-scolaires passés par des élèves dans les années 1960 (cf. encadré 4).

\footnotetext{
3. Pour concilier cette prise en compte des cohortes avec l'analyse de fratrie, il était nécessaire que les deux membres d'une fratrie appartiennent à la même cohorte. Nous avons pour cela attribué aux deux frères et soeurs la cohorte du plus jeune. Quatre cohortes (25-34 ans, 35-44 ans, 45-54 ans, 55-65 ans en 2003) ont ainsi été créées, de taille suffisante pour pouvoir mener des analyses sur chacune d'elles: elles contiennent respectivement 7 800, 9 130, 7890 et 5134 individus.
}

Encadré 4

\section{DES RÉSULTATS CONFIRMÉS À L'AIDE DE L'ENQUÊTE INED DE 1965 SUR LE NIVEAU INTELLECTUEL DES ENFANTS}

Reconstituer les fratries dans l'enquête Ined de 1965 sur le niveau intellectuel des enfants d'âge scolaire

L'enquête sur le niveau intellectuel des enfants d'âge scolaire réalisée en 1965 par l'Ined auprès d'élèves âgés de 6 à 14 ans comporte près de 120000 observations au total. Le fichier public ne permet toutefois pas de savoir si certains des élèves interrogés appartenaient à la même famille. Grâce au travail de F. Moreau (Ined), 6608 fratries d'au moins deux individus ont pu être identifiées, dont 358 couples de jumeaux. Cela a notamment été rendu possible par l'utilisation d'une variable conservée dans les fichiers d'archive, qui renseigne pour chaque établissement scolaire la position du patronyme de l'enquêté dans l'ordre alphabétique. Cette enquête possède sur l'enquête $F Q P$ l'avantage de ne pas être limitée à des variables renseignées pour au plus deux membres d'une fratrie. En contrepartie, elle présente deux inconvénients: d'une part son ancienneté, notamment en raison des changements importants ayant affecté le système scolaire depuis les années 1960, d'autre part le caractère imparfait de la méthode utilisée pour reconstituer les familles, qui ne capte notamment que les fratries dont les membres étudient dans le même établissement scolaire. Le biais qu'implique ce second inconvénient n'est pas négligeable si l'on 
L'estimation, réalisée à partir de modèles multiniveaux, permet de retrouver les mêmes ordres de grandeur que ceux obtenus grâce à l'enquête
FQP. Cela confirme l'idée d'une faible évolution de l'influence familiale cohésive au fil du temps.

Encadré 4 (suite)

considère que la mixité des établissements n'était pas encore généralisée à cette époque.

La variable d'intérêt utilisée est le résultat des élèves à des tests dits "d'intelligence ". Ces tests, étalonnés pour être comparables malgré les différences de niveaux séparant les élèves selon leur classe, sont interprétés comme reflétant les aptitudes scolaires des élèves. Ils correspondent d'ailleurs en moyenne au jugement de leurs professeurs, également recueillis dans l'enquête.

\section{Les modèles multiniveaux, plus adaptés pour prendre en compte les fratries de plus de deux individus}

Nous avons utilisé des modèles multiniveaux, ou modèles linéaires hiérarchiques, qui permettent de mesurer l'importance respective de différents niveaux imbriqués les uns dans les autres. Ces modèles sont conceptuellement équivalents aux modèles Mimic, en ce qu'ils permettent de mesurer la part de variance expliquée par un «niveau » ou "facteur ", qui est ici la famille. Hox et al. (1996) soulignent qu'en présence de données comportant des renseignements sur plus de deux individus dans la fratrie, l'approche par les modèles multiniveaux est préférable. Bien que les modèles multiniveaux diffèrent des modèles Mimic dans leurs modalités d'estimation, ils conduisent à des résultats très proches de ceux obtenus avec ces derniers lorsqu'ils sont utilisés sur les mêmes données.

Dans le cadre des modèles de fratrie, le premier niveau est le niveau individuel, et le deuxième niveau est le niveau familial. Les modèles multiniveaux permettent alors, au moyen des parts de variance expliquée, de déterminer l'influence de chaque niveau sur une variable d'intérêt, ici, le score obtenu au test. Ces modèles sont décrits notamment par Snijders et Bosker (1999). Le modèle vide à deux niveaux correspond aux deux équations :

Niveau 1 (individuel) : $Y_{\mathrm{ij}}=\alpha_{\mathrm{j}}+u_{\mathrm{ij}}$

Niveau 2 (familial) : $\alpha_{\mathrm{j}}=\beta+v_{\mathrm{j}}$

$\alpha_{i}$ représente la moyenne pour la variable $Y$ au sein de la fratrie j et $u_{i j}$ les variations individuelles autour de cette moyenne. $\beta$ représente alors la moyenne de la variable $Y$ parmi l'ensemble des fratries considérées, $v_{\mathrm{i}}$ reflétant les variations de chaque fratrie autour de cette moyenne. La variance des scores obtenus peut ainsi être décomposée en deux parties: une partie intra-familles (within) et une partie inter-familles (between). Le rapport entre la variance inter-familles et la variance totale reflète alors l'impact total de la famille sur les scores obtenus. Ce rapport est appelé coefficient de corrélation intra-classe (ICC).

\section{Des résultats similaires à ceux obtenus avec l'enquête FQP}

L'influence familiale cohésive s'élève à $51,1 \%$, ce qui signifie que la moitié des disparités de scores de compétences scolaires peut être imputée à une influence de la famille commune aux membres de la fratrie. Le résultat est très similaire à celui que nous avons observé à l'aide de l'enquête $F Q P$ pour le plus haut diplôme obtenu, alors même que nous raisonnons ici sur des tests de compétences passés dans le jeune âge. La différence entre les deux enquêtes est également temporelle, puisque l'enquête Ined concerne des tests tous passés la même année, il y a cinquante ans. L'enquête $F Q P$ comprend au contraire des informations sur des diplômes obtenus sur une longue période.

Les variables renseignées individuellement et caractérisant la famille, comme le groupe professionnel du chef de famille ou le fait d'habiter dans la même commune que celle de l'établissement scolaire, et la configuration de fratrie, comme la taille de la fratrie et le fait d'être aîné, ne permettent de rendre compte que de $12 \%$ de la variance des scores de compétences scolaires : $39 \%$ de la variabilité des scores de compétences relève donc d'une influence familiale cohésive inexpliquée.

L'influence familiale cohésive est plus élevée dans le cas de jumeaux. Elle vaut alors $73 \%$, ce qui laisse penser que la socialisation fraternelle, qui joue déjà un rôle important dans le cas des fratries non gémellaires, revêt pour les jumeaux une importance particulière. Les parents peuvent également avoir des pratiques éducatives qui favorisent spécialement la similarité de leur parcours. Cela résulte notamment du fait qu'ils ont le même âge et qu'ils sont donc traités de manière semblable. L'influence cohésive très élevée résulte aussi du fait que l'on avait moins tendance dans les années 1960 à séparer et à différencier les jumeaux. Enfin, on peut supposer que l'influence de facteurs génétiques soit particulière pour des jumeaux monozygotes, qui partagent à la naissance le même génome. Cette influence ne peut toutefois pas être mesurée indépendamment des multiples expériences de socialisation, qui sont plus ou moins partagées par les jumeaux ou membres de la fratrie. 


\section{Moins importante sur la profession que sur le diplôme, l'influence familiale n'est expliquée que pour moitié par les caractéristiques familiales observées}

Dans le cas où la variable d'intérêt est le score de la profession, l'analyse de l'influence familiale attribue au facteur familial 31,9\% de la variabilité de la place au sein de la hiérarchie des professions (cf. tableau 2). L'influence familiale cohésive exercée sur le statut professionnel est donc plus réduite que celle exercée sur le nombre d'années d'études. En effet, les facteurs qui interviennent après la fin des études et différencient les carrières des membres d'une même fratrie sont nombreux et souvent extérieurs à la sphère familiale. Ce constat ressort également de l'analyse de Sieben (2001), pour qui le facteur familial explique $37 \%$ de la variance du statut professionnel. L'inclusion des caractéristiques individuelles dans le modèle complet n'apporte là encore que peu de pouvoir

\footnotetext{
Tableau 2

Coefficients standardisés et parts de variance expliquées d'un modèle Mimic expliquant le score de la profession, incluant caractéristiques familiales puis caractéristiques individuelles et familiales

\begin{tabular}{|c|c|}
\hline & Score non linéaire \\
\hline \multicolumn{2}{|l|}{ Déterminants de la variable latente - variables familiales } \\
\hline Score de la PCS du père & $0,227\left(^{\star \star \star}\right)$ \\
\hline Score de la PCS de la mère & $0,156\left(^{* \star *}\right)$ \\
\hline Père inactif n'ayant jamais travaillé & $-0,004$ \\
\hline Mère inactive n'ayant jamais travaillé & $-0,001$ \\
\hline Score de la PCS du grand-père maternel & $0,100\left({ }^{\star \star \star}\right)$ \\
\hline Score de la PCS du grand-père paternel & $0,060\left(^{\star \star \star}\right)$ \\
\hline PCS du grand-père maternel manquante & $-0,090\left({ }^{\star \star \star}\right)$ \\
\hline PCS du grand-père paternel manquante & $-0,087(* \star *)$ \\
\hline Nombre d'années d'études du père & $0,128\left(^{* \star *}\right)$ \\
\hline Nombre d'années d'études de la mère & $0,047(* \star)$ \\
\hline Nombre d'enfants dans la fratrie & $-0,121\left({ }^{* * *}\right)$ \\
\hline Interaction avec une indicatrice " père cadre ou chef d'entreprise » & $0,040\left(^{\star \star \star}\right)$ \\
\hline Interaction avec une indicatrice « père ouvrier ou employé » & $-0,123\left({ }^{* \star *}\right)$ \\
\hline Divorce ou séparation des parents pendant les études & $-0,053\left({ }^{\star \star \star}\right)$ \\
\hline Parents résidant en Île-de-France à la fin des études & $0,129(* *)$ \\
\hline Taux de chômage dans la région des parents à la fin des études & $-0,001$ \\
\hline Score PCS manquant pour le père (hors inactif, jamais travaillé) & $-0,023(* *)$ \\
\hline Score PCS manquant pour la mère (hors inactive, jamais travaillé) & $-0,011$ \\
\hline Part de variance expliquée par le facteur familial latent (en \%) & 31,9 \\
\hline Part de la variance du facteur familial expliquée par les variables familiales observables (en \%) & 51,8 \\
\hline \multicolumn{2}{|l|}{ Déterminants de la variable dépendante - variables individuelles : } \\
\hline Sexe féminin & $-0,103\left({ }^{\star \star \star}\right)$ \\
\hline Aîné & $0,043\left(^{* * *}\right)$ \\
\hline Benjamin & $-0,008$ \\
\hline Âge de la mère à la naissance & $0,047\left({ }^{* \star *}\right)$ \\
\hline Effet du facteur familial sur le score de profession & $0,561(* \star *)$ \\
\hline Part de variance expliquée par le facteur familial latent et les variables individuelles (en \%) & 33,0 \\
\hline
\end{tabular}

Lecture : pour la question de la différence entre les coefficients du répondant et ceux du frère/sœur dans les équations expliquant le score, ainsi que pour le traitement des valeurs manquantes de la PCS de la mère, on se reportera au tableau 1.

Une augmentation du score de la PCS du père égale à un écart-type aurait conduit à une augmentation de 0,227 écart-type du facteur familial, lui-même positivement relié au score de profession du répondant $(0,561)$. La part de variance expliquée par le facteur familial latent est de $31,9 \%$

${ }^{* * *}$ : significatif au seuil de $1 \%$; ** : significatif au seuil de $5 \%$; * significatif au seuil de $10 \%$

Champ : personnes âgées de plus de 25 ans résidant en France métropolitaine, dont les parents résidaient en France au moment de la fin de leurs études.

Source : enquête FQP 2003, Insee.
} 
explicatif supplémentaire : la part expliquée de la variabilité du statut de la profession vaut alors 33,0 \%. De même que pour le nombre d'années d'études, le facteur familial n'est ici expliqué qu'à moitié $(51,8 \%)$ par les caractéristiques familiales observables, qui ne décrivent donc que partiellement l'influence familiale commune aux membres de la fratrie ${ }^{4}$.

Les coefficients de ce modèle complet sont donnés dans le tableau 2. Le score de profession des parents gagne en importance pour expliquer celui de la profession des enfants, tandis que le nombre d'années d'études des parents devient moins déterminant, en particulier celui de la mère. Concernant les caractéristiques individuelles, si le fait d'être une fille exerçait un effet positif sur le nombre d'années d'études, il exerce au contraire ici un effet significativement négatif. De même que pour l'influence familiale sur le niveau d'études, on n'observe pas d'évolution significative de l'influence familiale sur la profession au fil des cohortes.

L'utilisation de modèles Mimic appliqués aux données de fratrie disponibles dans l'enquête FQP 2003 permet ainsi d'établir une décomposition de la variance des parcours de la population considérée qui met en évidence une mesure globale de l'influence familiale cohésive plus forte pour le niveau d'études que pour le score de la profession. Pour ces deux étapes du parcours de l'individu, l'influence familiale mesurée repose pour moitié environ sur des caractéristiques qui sont inobservables dans les enquêtes standard (cf. figure II).

\section{L'influence familiale cohésive est plus forte pour les binômes fraternels de même sexe, lorsque l'écart d'âge entre frères et sours est limité ou les diplômes des parents plus homogènes}

L'influence familiale cohésive, qui s'exerce plus fortement sur les niveaux d'éducation qu'en matière de profession, peut par ailleurs être plus ou moins prononcée pour certaines catégories de population. Il est ainsi intéressant d'effectuer la mesure de l'influence familiale cohésive sur différents sous-ensembles de l'échantillon, en fonction de certaines caractéristiques du binôme fraternel ou encore des parents.

L'influence familiale apparaît ainsi plus forte pour les binômes de même sexe issus d'une même fratrie que parmi les binômes de sexes différents (et plus forte pour les binômes composés de deux sœurs que pour ceux composés de deux frères), et ce pour le niveau scolaire comme pour la position dans la hiérarchie des professions : l'influence familiale cohésive explique ainsi $60 \%, 56 \%$ et $52 \%$ du niveau d'études, pour les binômes de deux sœurs, de deux frères et les binômes mixtes respectivement ; et $39 \%$, $35 \%$ et $29 \%$ dans le cas de la position sociale.

\footnotetext{
4. Ces résultats ne changent pas lorsqu'on utilise l'échelle de position socio-scolaire de Le Donné et Rocher (2010), qui par sa construction diffère fortement de l'échelle de prestige de Chambaz et al. puisqu'elle attribue à chaque PCS une mesure de sa proximité avec le système socio-scolaire. Notamment, la part de variance expliquée par le facteur familial est dans ce cas de 36,5\%, 49,7 \% de celle-ci étant elle-même expliquée par les variables observables.
}

Figure II

Décomposition de l'influence familiale cohésive

\begin{tabular}{|c|c|c|c|}
\hline Niveau d'études & $26 \%$ & $28 \%$ & $46 \%$ \\
\hline $\begin{array}{c}\text { Prestige } \\
\text { de la profession }\end{array}$ & $17 \%$ & $15 \%$ & $68 \%$ \\
\hline
\end{tabular}

Lecture : l'influence familiale cohésive est décomposée entre l'effet des caractéristiques familiales observables dans l'enquête FQP et la part inobservable de l'influence familiale cohésive. $54 \%$ des variations des parcours individuels en termes de niveau d'études sont expliquées par le facteur familial latent, et relèvent à ce titre de l'influence familiale cohésive. La moitié de cette influence, à savoir $26 \%$ de la variance totale, est expliquée par les variables renseignées dans l'enquête FQP. L'autre moitié, $28 \%$, renvoie à des caractéristiques familiales inobservables. Enfin, $46 \%$ des variations de ces parcours individuels ne relève pas de l'influence familiale cohésive, ce qui comprend notamment l'effet de caractéristiques individuelles qui diffèrent selon les membres de la fratrie.

Champ : Personnes âgées de plus de 25 ans résidant en France métropolitaine, dont les parents résidaient en France au moment de la fin de leurs études

Source : enquête FQP 2003, Insee. 
Le fait que la socialisation parentale soit différente selon le genre de l'enfant participe de ce résultat, d'une part en différenciant le processus d'orientation selon le genre (Duru-Bellat, 1999), et d'autre part parce que les transmissions parentales sont fondées sur des identifications entre parents et enfants souvent sexuées (Henri-Panabière, 2010). L'école participe également à une socialisation différenciée selon le genre (Duru-Bellat, 1999). Ce résultat pourrait par ailleurs trouver une explication dans une socialisation fraternelle qui serait plus forte entre les membres de fratries du même sexe.

L'influence familiale cohésive sur le nombre d'années d'études est également moindre lorsque l'écart d'âge dans la fratrie est plus grand. De $59 \%$ pour un écart d'âge inférieur ou égal à 5 ans, elle diminue à $51 \%$ pour un écart d'âge compris entre 6 et 9 ans et est de $44 \%$ lorsque l'écart d'âge est compris entre 10 et 15 ans. L'importance de l'écart d'âge pourrait ici à la fois s'expliquer par une socialisation fraternelle facilitée par la proximité en âge et par une évolution de l'environnement familial et des caractéristiques de l'éducation parentale au cours du temps qui rend d'autant plus dissemblable la socialisation reçue par les frères et sœurs qu'ils sont séparés par un écart d'âge important.

Enfin, l'importance de l'influence familiale cohésive est dans une certaine mesure différenciée selon l'hétérogénéité des diplômes des parents. L'influence familiale explique ainsi $56 \%$ des parcours scolaires dans les binômes fraternels dont les parents ont fait le même nombre d'années d'études contre $46 \%$ dans les binômes dont les parents ont une différence de niveau d'études comprise entre 1 an et 2 ans. Ce pourcentage s'établit à $42 \%$ seulement lorsque l'écart d'éducation est plus élevé. Ce résultat, qui repose sur l'échelle médiane pour le nombre d'années d'études, doit être lu avec précaution car les écarts diminuent dès lors que l'on emploie l'échelle modale. Il tend toutefois à montrer que les disparités intrafamiliales en termes de capital culturel participent d'une moindre ressemblance des parcours dans la fratrie, conformément à ce que montrent les enquêtes qualitatives de Lahire (1995). Cela résulterait d'une moindre cohérence des dispositions transmises du point de vue de la culture scolaire légitime.

L'influence familiale cohésive, agissant selon des mécanismes de socialisation horizontale comme verticale, varie donc selon les caractéristiques de la fratrie et plus généralement de la famille. Sa mesure est dans tous les cas largement plus exhaustive que celle résultant des seules caractéristiques observables présentes dans les enquêtes. Elle permet en effet de capter l'impact de caractéristiques familiales inobservées, dont l'omission conduirait à sous-estimer de moitié l'importance de l'influence familiale aussi bien sur l'éducation que sur la profession. Cette mesure peut ainsi être utilisée comme outil permettant de contrôler de façon plus complète les déterminants familiaux des parcours des individus, lorsque ceux-ci représentent un potentiel biais d'endogénéité.

\section{En contrôlant par l'influence familiale cohésive plutôt que par les seules caractéristiques familiales observables, l'effet de l'éducation sur la profession se voit minoré d'un quart}

Les modèles Mimic permettent de mieux tenir compte des influences à l'œuvre entre les différentes variables, par rapport au schéma simplifié défini en première analyse (cf. figure III). Il est ainsi possible d'étudier l'effet de l'éducation sur la profession tout en contrôlant par l'influence cohésive de la famille, et non pas simplement par des caractéristiques observables. La partie du biais de variable omise ainsi éliminée correspond aux caractéristiques inobservées captées par le facteur familial latent ${ }^{5}$. Ces caractéristiques peuvent avoir trait aux compétences individuelles, mais également à des éléments liés à l'origine sociale sans relever des compétences : à compétences données, un individu issu d'une famille plus favorisée pourra être incité à poursuivre plus longtemps ses études. Il pourra également bénéficier du capital social de ses parents pour s'établir plus facilement dans la vie professionnelle.

Le modèle inclut les influences représentées sur la figure III, en particulier celle des caractéristiques individuelles, de l'éducation et du facteur familial latent sur la profession. L'échelle modale est retenue pour l'éducation et l'échelle non linéaire de statut social pour la profession. Les caractéristiques familiales et individuelles observables sont les mêmes que dans les modèles Mimic précédents. Le paramètre d'intérêt est le coefficient non standardisé correspondant à l'influence de l'éducation du répondant sur la profession du répondant: celui-ci, significatif au seuil de $1 \%$, est de 0,127 . Une année

5. Le biais de variable omise qui pourrait subsister correspondrait ainsi à des différences d'habileté innées mais non liées à l'appartenance familiale. 
d'études supplémentaire fait ainsi augmenter de 0,127 le score de statut social associé à la profession exercée (sur une échelle allant de $-1,52$ à 2,19). Le coefficient équivalent dans une régression linéaire classique contrôlant par toutes les caractéristiques familiales et individuelles observables est 0,164 , également significatif. Le fait de contrôler uniquement par les caractéristiques familiales observables conduit ainsi à surestimer l'influence de l'éducation sur le score de la profession de plus de $25 \%$.

Ces effets sont estimés sur l'ensemble de la population, toutes cohortes confondues. Les résultats cohorte par cohorte sont présentés dans le tableau 3.

La progression observée de l'effet propre de l'éducation ne conduit pas à une conclusion tranchée, car elle recouvre à la fois un effet d'âge (lorsque l'âge augmente, et donc aussi le temps écoulé entre la fin des études et le dernier emploi occupé, l'influence directe de l'éducation sur l'activité professionnelle diminue) et un effet de génération (réelle augmentation de l'effet propre au fil des générations). La cohorte la plus jeune, pour laquelle la profession renseignée est la plus proche temporellement de la première profession exercée à la sortie des études, connaît un écart entre les deux coefficients (effet pur et effet capté par régression linéaire avec contrôles) plus réduit que pour l'ensemble de la population. Même dans ce cas, on surestime de plus de $20 \%$ l'effet de l'éducation sur le statut de la profession si l'on ne tient pas compte de l'influence cohésive de la famille d'origine.

\section{Une forte influence familiale cohésive indique une reproduction sociale ou une mobilité sociale de la fratrie}

Les modèles de fratrie que nous avons utilisés dans ce qui précède sont apparus dans la lignée des questionnements sur la mobilité sociale, à la suite des critiques adressées aux analyses

\section{Figure III}

Modèle Mimic permettant de mesurer l'effet du niveau d'études sur la profession

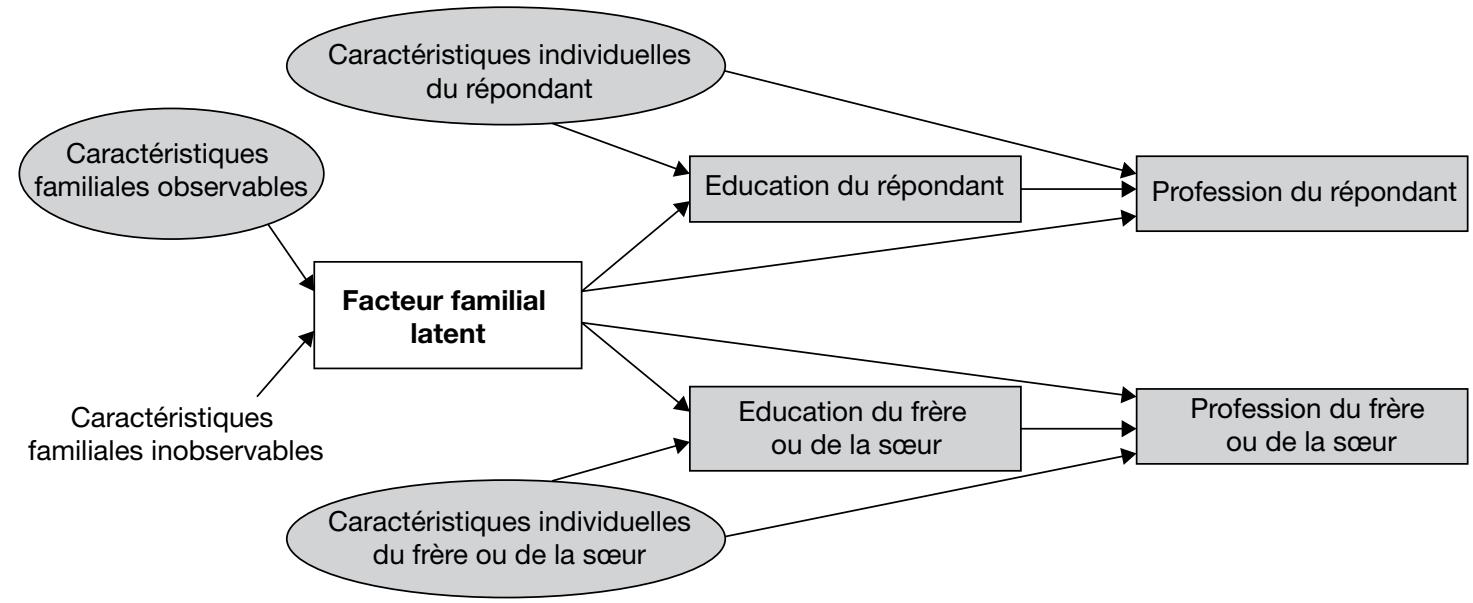

Lecture : les flèches représentent des relations de causalité modélisées entre des variables présentes dans l'enquête ou estimées.

Tableau 3

Effet du nombre d'années d'études sur le score de la profession

\begin{tabular}{|c|c|c|}
\hline Cohorte & Modèle Mimic & Régression linéaire \\
\hline 34 ans et moins & 0,160 & 0,197 \\
35 à 44 ans & 0,146 & 0,198 \\
45 à 54 ans & 0,135 & 0,178 \\
55 ans et plus & 0,116 & 0,156 \\
\hline
\end{tabular}

Lecture : parmi les individus de l'échantillon âgés de 34 ans et moins, on mesure au moyen d'un modèle Mimic qu'une année supplémentaire d'études fait augmenter de 0,160 le score de statut social.

Champ : personnes âgées de plus de 25 ans résidant en France métropolitaine, dont les parents résidaient en France au moment de la fin de leurs études.

Source : enquête FQP 2003, Insee. 
« verticales » étudiant le lien entre les parcours des individus et ceux de leurs parents, notamment au moyen de tables de mobilité.

L'information fournie par les modèles de fratrie ne peut toutefois pas à elle seule fournir une information exhaustive portant sur l'influence familiale : en effet, une proximité des parcours au sein de la fratrie peut correspondre aussi bien à des situations de reproduction sociale, qu'à des cas de mobilité sociale pour l'ensemble de la fratrie. Dans le premier cas, la distance verticale entre parents et enfants apparaît plus réduite, et plus élevée dans le second cas.

Pour approfondir l'interprétation que nous avons faite de l'influence familiale à l'aide des modèles Mimic, on peut dresser une typologie simplifiée des parcours familiaux prenant à la fois en compte la fratrie et la lignée parent-enfant. On obtient trois cas-types :

- la reproduction sociale, caractérisée par une proximité des parcours au sein de la fratrie, mais également entre les membres de la fratrie et leurs parents ;

- la mobilité sociale de la fratrie, qui suppose quant à elle une proximité des parcours dans la fratrie, mais une certaine distance sociale par rapport aux parents ;

- la différenciation des parcours dans la fratrie.
Dans l'analyse de fratrie telle que nous l'avons menée avec les modèles Mimic, les deux premiers cas-types, reproduction sociale et mobilité de la fratrie, ne peuvent être différenciés dans la mesure où ils sont tous deux caractérisés par une forte influence familiale cohésive. L'analyse verticale de la mobilité sociale permet de distinguer ces deux groupes en attribuant un sens différent à la forme d'influence familiale qui les caractérise (cf. figure IV). Dans le cas de la reproduction sociale, les individus subissent une restriction de leurs possibilités de parcours du fait de leur appartenance à un milieu modeste, ou peuvent maintenir la position élevée de leur famille grâce aux dispositions qu'ils ont acquises par leurs parents. Dans le cas de la mobilité de la fratrie, les parents transmettent des dispositions à l'ensemble de leurs enfants, qui permettent à ces enfants d'accéder, lorsqu'il s'agit d'une mobilité ascendante, à un milieu plus élevé que le leur (notamment grâce à une réussite scolaire) $)^{6}$.

6. Les caractéristiques familiales qui permettent une mobilité sociale de l'ensemble de la fratrie, ou le maintien d'une position sociale élevée, sont difficiles à appréhender. Les travaux qualitatifs de Lahire sur des familles modestes dont certains des enfants connaissent une ascension sociale (1995) et d'Henri-Panabière (2010) sur des familles favorisées faisant face à un échec scolaire donnent des précisions à ce sujet. Dans des familles dont les parents ne détiennent pas des diplômes supérieurs, l'importance symbolique accordée au travail et à la réussite scolaire, ainsi que la présence à la maison peuvent favoriser une ascension sociale. Dans les familles favorisées, la transmission des dispositions scolaires aux enfants peut reposer également sur la présence à la maison, mais aussi sur l'ancienneté du capital culturel dans la famille.

Figure IV
Typologie des formes d'influence familiale selon les parcours familiaux

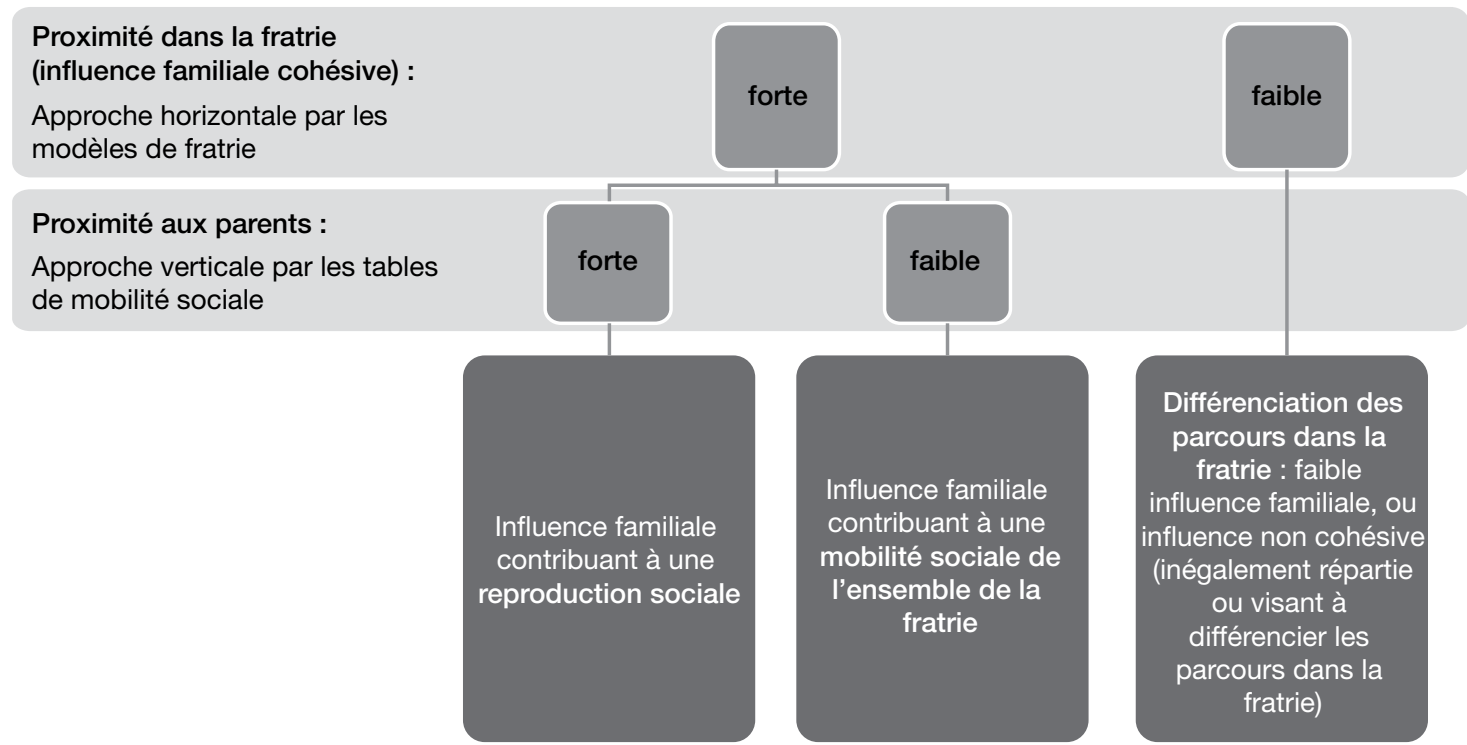

Lecture : en croisant les deux dimensions d'analyse des parcours que sont la proximité dans la fratrie (influence familiale cohésive) et la proximité sociale aux parents (reproduction ou mobilité sociale), on distingue trois types d'influence familiale. 
Le troisième cas-type fait état d'une hétérogénéité de la réussite scolaire et professionnelle dans la fratrie qui ne correspond à aucune des deux formes d'influence familiale que nous venons de décrire. Selon les modèles de fratrie, une hétérogénéité de réussite dans la fratrie reflète une faible influence familiale cohésive. Mais on peut au contraire supposer l'existence d'une influence familiale non cohésive, conduisant à des parcours différents dans la fratrie. Elle consisterait en une répartition inégale des ressources familiales entre membres de la fratrie ${ }^{7}$, ou en une influence familiale favorisant pour chacun le développement d'une identité personnelle distincte de celle des autres membres de la famille, parents comme enfants ${ }^{8}$.

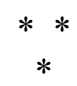

S'appuyer sur des données renseignées pour plusieurs membres d'une même fratrie permet de mesurer une influence familiale «cohésive », qui s'applique à tous les membres de la fratrie et contribue à rapprocher leurs parcours. On montre ainsi l'importance des déterminants familiaux inobservables dans les enquêtes, pour l'explication du niveau d'études aussi bien que de la profession. Qu'il s'agisse de la réussite scolaire ou de la position dans la hiérarchie des professions, ces caractéristiques expliquent la moitié de l'influence familiale cohésive mesurable au travers des modèles de fratrie, qui elle-même explique la moitié environ de la variabilité des niveaux d'études, et un tiers environ dans le cas de la profession. L'effet de la famille sur les parcours est donc nettement plus fort que celui que l'on observe à partir des seules caractéristiques individuelles liées à l'origine sociale.

En mesurant l'effet de la famille à l'aune de la proximité des parcours dans la fratrie, on assimile à l'influence familiale certaines caractéristiques communes aux membres de la fratrie mais qui ne relèvent toutefois pas directement de la socialisation parentale, et qui viendraient en renforcer ou en atténuer les effets. Ainsi la possibilité avancée plus haut que le lieu

7. Théorisée par Zarca (1995) et plus récemment étudiée par S. Gollac (2009) pour les familles d'indépendants.

8. Elle est notamment théorisée par De Singly (1996) et analysée par Kellerhals et Montandon (1991), qui montrent que ce type d'influence est plus répandu dans les classes supérieures, bien qu'elle entre en conflit avec l'exigence scolaire propre à garantir le maintien de la position sociale de la famille. d'habitation des parents soit un choix relevant d'une stratégie éducative ne peut être généralisée. À cet égard, la proximité des parcours pourrait relever de facteurs autres que le milieu familial (et même plus largement le milieu social d'origine) si l'on conçoit par exemple un établissement scolaire permettant à deux membres d'une même fratrie de s'émanciper de leur milieu social et familial d'origine. Malgré cette précaution - difficilement quantifiable - l'intérêt de la mesure de l'influence familiale cohésive est fort: celle-ci permet d'appréhender l'effet de caractéristiques familiales absentes des enquêtes, voire non mesurables, jusqu'aux moyens les plus informels de transmettre des dispositions socialement valorisables, aux attitudes corporelles et à d'éventuels facteurs génétiques dont l'effet est difficilement séparable de celui des autres formes d'hérédité familiale.

Cette mesure plus exhaustive de l'influence familiale est par ailleurs un instrument dans l'étude de relations entre des variables pouvant être chacune impactée par l'origine familiale. Ainsi, en contrôlant par l'influence familiale cohésive, on obtient un effet de l'éducation sur la profession minoré par rapport à celui obtenu en contrôlant par les seuls déterminants familiaux observables. Enfin, la mesure de l'influence familiale cohésive gagne à être articulée avec la notion plus classique de mobilité sociale, afin de distinguer les situations de reproduction sociale de celles où la mobilité sociale concerne toute la fratrie, ainsi que pour s'interroger sur l'existence de formes non cohésives d'influence familiale.

L'approfondissement de ces questions nécessiterait l'introduction plus répandue de données de fratrie dans les enquêtes de la statistique publique, ainsi que la conduite d'enquêtes qualitatives permettant d'analyser l'influence familiale au sein de fratries selon leur position dans l'espace social. On peut saluer, pour ce qui relève du premier point, la reconduction $\mathrm{du}$ module complémentaire concernant les fratries dans l'enquête FQP conduite sur les années 2014 et 2015. Quant aux enquêtes qualitatives, elles apportent un regard complémentaire sur la question des rapports entre influence familiale et diversité des parcours, par exemple en étudiant plus en détail les réussites hétérogènes dans la fratrie (Peugny, 2012) ou encore les effets sur la construction des parcours d'orientation dans la fratrie de normes familiales privilégiant l'excellence scolaire ou l'épanouissement individuel (Grobon, 2013). 


\section{BIBLIOGRAPHIE}

Blau P.M. et Duncan O.D. (1967), The American Occupational Structure, New-York, Wiley.

Bowles S. (1972), « Schooling and Inequality from Generation to Generation », Journal of Political Economy, vol. 80, n 3, pp. 219-251.

Chambaz C., Maurin E. et Torelli C. (1998), «L'évaluation sociale des professions en France. Construction et analyse d'une échelle des professions », Revue française de sociologie, vol. 39, $\mathrm{n}^{\circ} 1$, pp. 177-226.

Darmon M. (2001), «La socialisation, entre famille et école : observation d'une classe de première année de maternelle », Sociétés \& représentations, $\mathrm{n}^{\circ} 11$, pp. 517-538.

De Graaf P. M. (1986), « The Impact of Financial and Cultural Resources on Educational Attainment in the Netherlands », Sociology of Education, vol. $59, \mathrm{n}^{\circ} 4$, pp. 237-246.

De Singly F. (1996), Le Soi, le couple et la famille, Paris, Nathan.

Duru-Bellat M. (1999), « Les choix d'orientation : des conditionnements sociaux à l'anticipation de l'avenir », dans Lemel Y. et Roudet B., Filles et garçons à l'adolescence, socialisations différentielles, L’Harmattan, pp. 117-150.

Ganzeboom H.B.G. et Treiman D. (1993), « Preliminary Results on Educational Expansion and Educational Achievement in Comparative Perspective », dans Becker H. et Hermkens P., Solidarity of Generations: Demographic, Economic and Social Change, and Its Consequences, Thesis Publishers, Amsterdam, pp. 467-506.

Gollac S. (2009), « Travail indépendant et transmissions patrimoniales: le poids des inégalités au sein des fratries ", Économie et Statistique, $\mathrm{n}^{\circ}$ 417-418, pp. 56-75.

Griliches Z. (1979), « Sibling Models and Data in Economics: Beginnings of a Survey », Journal of Political Economy, vol. 87, n 5, pp. 37-64.

Griliches Z. et Mason W.M. (1972), « Education, Income and Ability », Journal of Political Economy, vol. 80, $\mathrm{n}^{\circ} 3$, pp. 74-103.

Grobon S. (2013), «Le social dans la fratrie, entre ressemblance familiale et différenciation individuelle », Dossiers d'études de la Caisse nationale des Allocations familiales (CNAF), $\mathrm{n}^{\circ} 161$, pp. 3-147.

Hauser R.M. et Sewell W.H. (1977), « On the effects of family structure on achievements », dans Taubman P.J., Kinometrics: The Determinants of Educational Attainment, Mental Ability, and Occupational Success Within and Between Families, North-Holland, Amsterdam, pp. 255-283.

Hauser R.M. et Wong R. (1989), «Sibling Resemblance and Inter-Sibling Effects in Educational Attainment», Sociology of Education, vol. $62, \mathrm{n}^{\circ} 3$, pp. 149-171.

Henri-Panabière G. (2010), « Élèves en difficultés issus de parents fortement diplômés : une mise à l'épreuve empirique de la notion de transmission culturelle », Sociologie, vol. 1, n 4, pp. 457-478.

Hox J.J., Dronkers J., Evans M. et Kelley J. (1996), «A comparison of some multilevel sibling models », dans Ernste H. (Ed.), Multilevel Analysis with Structural Equations Models, ETH Zürich, pp. 19-28.

Jöreskog K. et Goldberger A. (1975), « Estimation of a model with multiple indicators and multiple causes of a single latent variable », Journal of the American Statistical Association, vol. 70, $\mathrm{n}^{\circ}$ 351, pp. 631-639.

Kellerhals J. et Montandon C. (1991), Les stratégies éducatives des familles. Milieu social, dynamique familiale et éducation des préadolescents, Delachaux et Niestlé, Genève.

Lahire B. (1995), Tableaux de Famille. Heurs et malheurs scolaires en milieux populaires, Gallimard/Seuil, Paris.

Le Donné N. et Rocher T. (2010), «Une meilleure mesure du contexte socio-éducatif des élèves et des écoles ", Éducation et Formations, n 79 , pp. 103-115.

Merllié D. et Monso O. (2007), «La destinée sociale varie avec le nombre de frères et sœurs », France, Portrait social, Insee, pp. 135-153.

Monso O. et Thévenot L. (2008), « Des milieux sociaux au marché du travail: les questionnements sur la société française pendant quarante ans d'enquêtes «Formation et Qualification 
Professionnelle », Document de travail du Crest, $\mathrm{n}^{\circ} 2008-20$, Insee.

Peugny C. (2012), «L'expérience vécue de la mobilité sociale : le poids de la fratrie », Informations sociales, $\mathrm{n}^{\circ} 173$, pp. 94-101.

Sieben I. (2001), Sibling Similarities and Social Stratification, Université de Nimègue, Hollande.

Sieben I. et De Graaf P.M. (2003), « The total impact of the family on educational attainment: a comparative sibling analysis ", European Societies, vol. 5, n 1 , pp. 33-68.

Vallot F. (1973), «Résultats globaux : niveau intellectuel selon le milieu social et scolaire. Enquête nationale sur le niveau intellectuel des enfants d'âge scolaire ", Travaux et Documents, $n^{\circ} 64$ Ined/PUF, Paris.

Van Eijck C.J.M. (1996), Family and Opportunity, a Sibling Analysis of the Impact of Family Background on Education, Occupation, and Consumption, Tilburg University Press, Tilburg, Hollande.

Zarca B. (1995a), «L'héritage et la mobilité sociale au sein de la fratrie I - L'héritage et la mobilité différentielle des frères », Population, vol. 50, n², pp. 331-356.

Zarca B. (1995b), «L'héritage et la mobilité sociale au sein de la fratrie II - L'activité professionnelle et la mobilité professionnelle des sœurs », Population, vol. 50, $\mathrm{n}^{\circ}$ 4-5, pp. 1137-1154. 
Tableau A

Échelles d'éducation

\begin{tabular}{|c|c|c|}
\hline Diplôme & Échelle 1 : médiane & Échelle 2 : mode \\
\hline $2^{\mathrm{e}}$ ou $3^{\mathrm{e}}$ cycle universitaire & 18 & 18 \\
\hline Grande école, diplôme d'ingénieur & 18 & 17 \\
\hline $1^{\text {er }}$ cycle universitaire & 16 & 17 \\
\hline BTS, DUT & 15 & 15 \\
\hline Paramédical ou social avec baccalauréat général & 16 & 16 \\
\hline Paramédical ou social sans baccalauréat général & 15 & 16 \\
\hline Baccalauréat général et diplôme technique secondaire & 14 & 14 \\
\hline Baccalauréat général seul & 14 & 14 \\
\hline Baccalauréat technologique ou professionnel et brevet professionnel & 14 & 14 \\
\hline BEI, BEC, BEA & 12 & 12 \\
\hline CAP, BEP et BEPC & 12 & 12 \\
\hline CAP, BEP seul & 11 & 11 \\
\hline BEPC seul & 12 & 12 \\
\hline CEP & 9 & 8 \\
\hline Aucun diplôme & 10 & 10 \\
\hline
\end{tabular}

Lecture : échelle 1 : nombre d'années d'études médian dans la catégorie de diplôme considérée. Échelle 2 : nombre d'années d'études modal dans la catégorie de diplôme considérée.

Champ : personnes âgées de plus de 25 ans résidant en France métropolitaine, dont les parents résidaient en France au moment de la fin de leurs études

Source : enquête FQP 2003, Insee.

Tableau B

Scores professionnels (échelles de Chambaz, Maurin et Torelli, 1998)

\begin{tabular}{|c|c|c|}
\hline PCS en 16 postes & Échelle linéaire & Échelle non linéaire \\
\hline Agriculteurs & $-0,29$ & $-0,43$ \\
\hline Artisans & $-0,03$ & 0,17 \\
\hline Commerçants et assimilés & 0,07 & 0,27 \\
\hline Chefs d'entreprise de 10 salariés ou plus & 0,54 & 1,18 \\
\hline Professions libérales & 1,03 & 2,19 \\
\hline Cadres de la fonction publique, professions intellectuelles et artistiques & 0,57 & 1,33 \\
\hline Cadres d'entreprise & 0,72 & 1,65 \\
\hline $\begin{array}{l}\text { Professions intermédiaires de l'enseignement, de la santé, de la fonction } \\
\text { publique et assimilés }\end{array}$ & 0,41 & 1,02 \\
\hline Professions intermédiaires administratives et commerciales des entreprises & 0 & 0 \\
\hline Techniciens & 0,31 & 0,78 \\
\hline Employés de la fonction publique & $-0,34$ & $-0,52$ \\
\hline Employés administratifs d'entreprise & $-0,07$ & 0,03 \\
\hline Employés de commerce & $-0,68$ & $-1,18$ \\
\hline Personnels des services directs aux particuliers & $-0,68$ & $-1,17$ \\
\hline Ouvriers qualifiés & $-0,32$ & $-0,50$ \\
\hline Ouvriers non qualifiés & $-0,80$ & $-1,52$ \\
\hline
\end{tabular}

Lecture : le score professionnel attribué aux agriculteurs selon l'échelle linéaire définie par Chambaz, Maurin et Torelli (1998) est de -0,29. Il est de - 0,43 selon leur échelle non-linéaire.

Source : Chambaz, Maurin et Torelli (1998). 
Tableau C

Statistiques descriptives portant sur les variables explicatives des modèles Mimic

\begin{tabular}{|c|c|c|c|}
\hline & Minimum & Moyenne & Maximum \\
\hline Score de la PCS du père & $-1,52$ & $-0,202$ & 2,19 \\
\hline Score de la PCS de la mère & $-1,52$ & $-0,447$ & 2,19 \\
\hline Père inactif n'ayant jamais travaillé & 0 & 0,001 & 1 \\
\hline Mère inactive n'ayant jamais travaillé & 0 & 0,314 & 1 \\
\hline Score de la PCS du grand-père maternel & $-1,52$ & $-0,368$ & 2,19 \\
\hline Score de la PCS du grand-père paternel & $-1,52$ & $-0,326$ & 2,19 \\
\hline PCS du grand-père maternel manquante & 0 & 0,308 & 1 \\
\hline PCS du grand-père paternel manquante & 0 & 0,335 & 1 \\
\hline Nombre d'années d'études du père & 8 & 10,3 & 18 \\
\hline Nombre d'années d'études de la mère & 8 & 10,1 & 18 \\
\hline Nombre d'enfants dans la fratrie & 2 & 4,00 & 19 \\
\hline Interaction avec une indicatrice « père cadre / chef d'entreprise » & 0 & 0,325 & 13 \\
\hline Interaction avec une indicatrice « père ouvrier ou employé » & 0 & 2,84 & 19 \\
\hline Divorce ou séparation des parents pendant les études & 0 & 0,067 & 1 \\
\hline Résidence en Île-de-France à la fin des études & 0 & 0,138 & 1 \\
\hline Taux de chômage dans la région des parents à la fin des études & 5,5 & 12,9 & 22,4 \\
\hline PCS manquante pour le père (hors inactif, jamais travaillé) & 0 & 0,003 & 1 \\
\hline PCS manquante pour la mère (hors inactive, jamais travaillé) & 0 & 0,013 & 1 \\
\hline Sexe féminin & 0 & 0,508 & 1 \\
\hline Aîné & 0 & 0,288 & 1 \\
\hline Benjamin & 0 & 0,345 & 1 \\
\hline Âge de la mère à la naissance & 11 & 27,7 & 58 \\
\hline
\end{tabular}

Lecture : les variables de nombre d'années d'études et de score de la profession pour les parents et les grands-pères sont respectivement l'échelle modale et l'échelle non linéaire.

Le score de la PCS du père de l'enquêté varie dans notre échantillon de -1,52 à 2,19. Sa valeur moyenne est de - 0,202.

Champ : personnes âgées de plus de 25 ans résidant en France métropolitaine, dont les parents résidaient en France au moment de la fin de leurs études.

Source : enquête FQP 2003, Insee. 
